\title{
Predicting the performance of cathodic protection systems with large scale interference
}

\author{
R. Adey ${ }^{1}$, J. M. W. Baynham ${ }^{1} \&$ T. Curtin ${ }^{2}$ \\ ${ }^{1}$ C M BEASY Ltd, Ashurst Lodge, Southampton, UK \\ ${ }^{2}$ Computational Mechanics Inc, Billerica, Ma, USA
}

\begin{abstract}
Understanding the interactions between cathodic protection systems is becoming more complex particularly in the marine and offshore industry. The search for oil and gas in deeper waters and remote environments has resulted in engineering designs with many components on the sea bed as well as at the sea surface. Traditional design methods are inadequate in these situations as interactions will occur between the Cathodic Protection (CP) systems of the components, and significant currents can develop in the connecting flow lines.

In this paper recent developments made in the BEASY Corrosion Modelling software are presented which enable these systems to be simulated. Examples are presented showing how computer modelling can play a major role in predicting the level of interaction and providing the key data for the design of the system and its integrity management.
\end{abstract}

\section{Introduction}

Boundary Element Methods (BEM) have been used to simulate the behaviour of cathodic protection systems since the late 1970s [1]. As the name implies, the method requires creation of elements, but only on the boundary (i.e. surfaces) of the problem geometry. The BEM is used to mathematically model the potential drop in the electrolyte represented by the Laplace equation. In many applications it is sufficient to couple these equations with the equations representing the electrochemical electrode kinetics on the metallic surfaces to form a system of equations capable of simulating the potential fields and current flowing in the electrolyte. 
It was common in the early models to make the assumption that the internal resistance of the structure (through which the current returns) is very small compared with that of the electrolyte and the electrode kinetics. Therefore it was possible to ignore the metal resistance when formulating the equations and hence there was no IR drop in the return path. Pipelines present a typical situation where this assumption is not valid as over a long pipeline there is significant drop in the potential due to the internal resistance of the pipeline metal. In new large scale deep water Oil and Gas developments not only are long pipelines present but there are hundreds of individual flow lines and connections each introducing a new electrical connection path which must be considered.

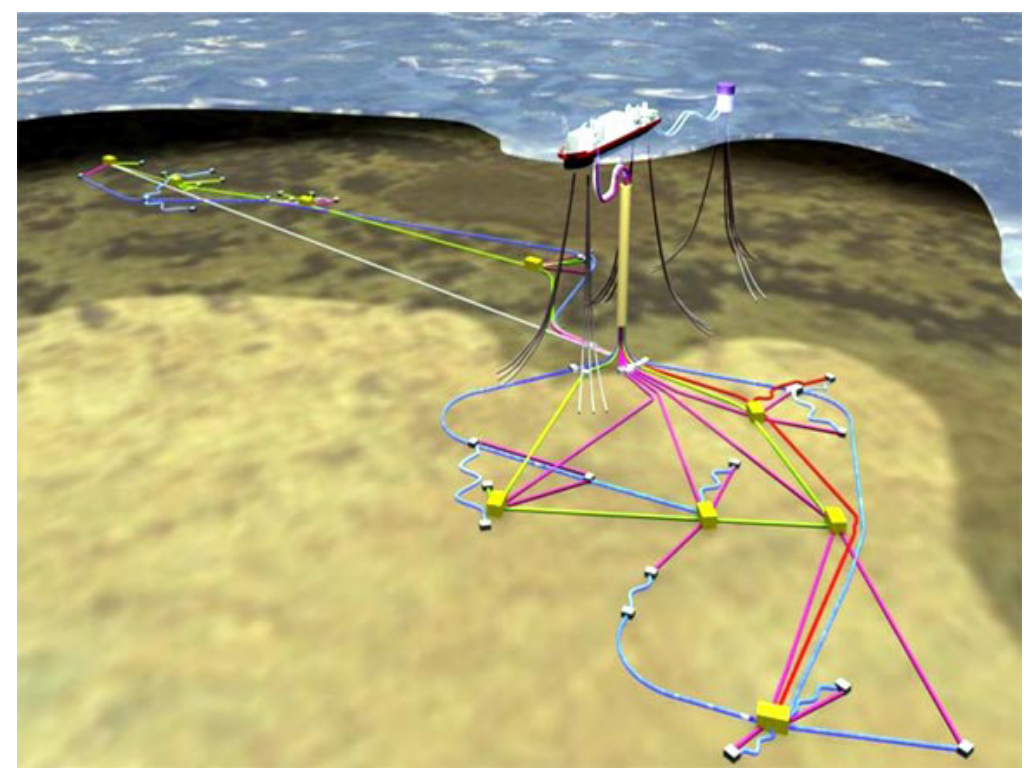

Figure 1: Example of a modern deepwater Oil and Gas development.

Standard BEM modelling tools are inadequate in these situations as interactions will occur between the Cathodic Protection (CP) systems, causing significant currents (and therefore voltage drops) to occur in the flow lines connecting the components. In this paper recent developments made in the BEASY Corrosion Modelling software are presented which enable these systems to be modelled.

\section{State of the art}

Numerical methods have been widely used in the corrosion field since the early 1980s when IMI Marstons employed them to help model the performance of the impressed anodes to be used on the Conoco TLP platform for the North sea [2]. 
They have been successfully compared with physical scale modeling experimental results and data obtained from tests performed on full size ships [3]. DeGiorgi et al. studied a 3D model of a U S Navy CG class ship [4] using boundary elements. The potential profiles for reference cell readings of -0.85 Volts $\mathrm{Ag} / \mathrm{AgCl}$ showed very good agreement between experimental and computational results. The difference between experimental and computational results was $6 \%$ for total current values, $5 \%$ for Amps to the propeller and $10 \%$ for Amps to the docking blocks. The general conclusions were that, boundary element methods are a viable technique for determining marine corrosion parameters.

Studies of interference between CP systems of a ship and nearby dock have been made [5], in which it was assumed the metal current paths had zero resistance.

\section{Internal resistances}

From a mathematical point of view the internal resistances can be viewed as a simple electrical circuit where the metal objects are connected by resistances or devices such as diodes. Figure 2

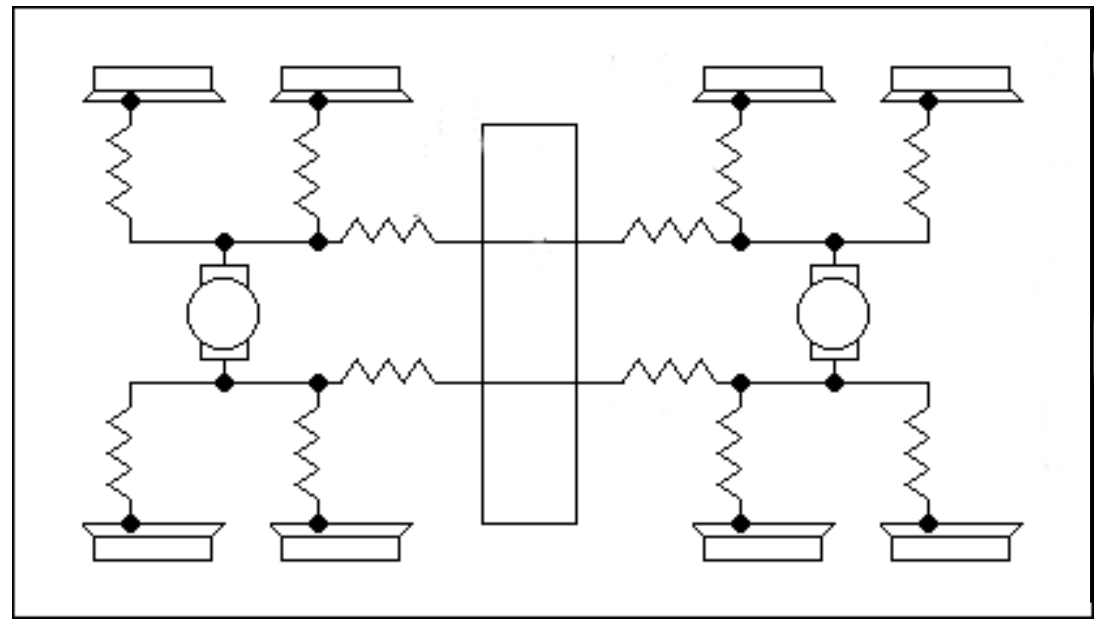

Figure 2: $\quad$ Example internal resistance circuit.

Each of the objects in the circuit represents a metal object being protected by a CP system. The resistances are the resistances of the connecting pipelines or flow lines connecting the different components in the structure.

If we consider a simple case of a FPSO connected to a buoy by an offloading line. The FPSO may be protected by an ICCP system and the buoy by a sacrificial anode system. If they are not electrically connected then there is unlikely to be interference as the only electrical return path is though the sea water itself. If they are connected by the offloading lines an electrical circuit is 
created which allows for example current to flow from the ICCP anodes on the FPSO to the buoy and current to return along the offloading lines.

This can be expressed mathematically as:

$$
I_{\text {FlowlineConnectedToBuoy }}=I_{\text {BuoyAnodes }}+I_{\text {BuoyMetalSurfaces }}
$$

Similarly for the FPSO

$$
I_{\text {FlowlineConnectedToFPSO }}=I_{\text {FPSOAnodes }}+I_{\text {FPSOMetalSurfaces }}
$$

For equilibrium the current flowing along the Flowline to either the buoy or the FPSO must balance.

The currents flowing into or out of the metal surfaces on the buoy and the FPSO can be determined by integrating the normal current density calculated by the boundary elements over the metal surfaces of the structure.

Therefore the internal circuit resistance equations and the boundary element equations representing the sea water can be solved together to predict the potentials and currents flowing in the system.

\subsection{User interface}

In a complex situation there may be hundreds of individual circuits and connections to be modelled. Therefore a Graphical User Interface (GUI) has been developed to simplify the task of the user in creating the model and to provide a visual means of auditing the connections.

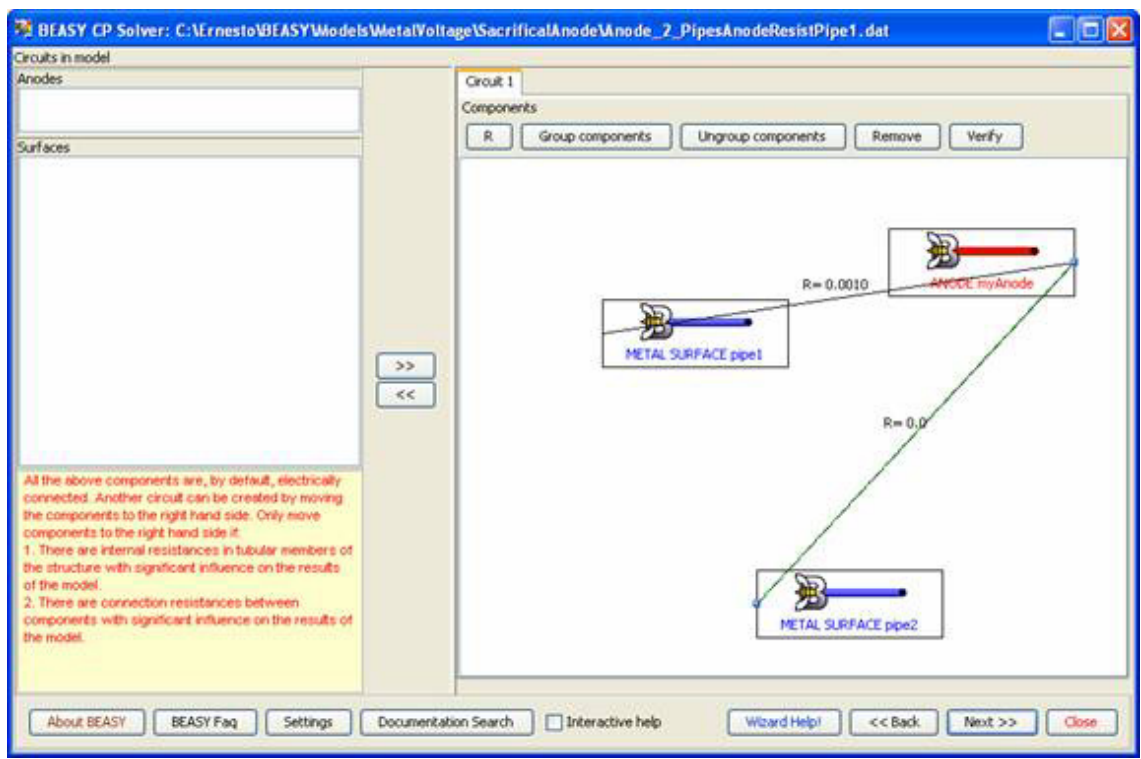

Figure 3: The Circuit editor enables electrical connections to be made between parts of the model and to specify their electrical resistance. 
Figure 3 shows a view of the circuit editor. Metal objects can be selected and electrical connections made simply by picking them with the mouse and specifying the type of connection. Structures can also be grouped together if their electrical connections have negligible resistance.

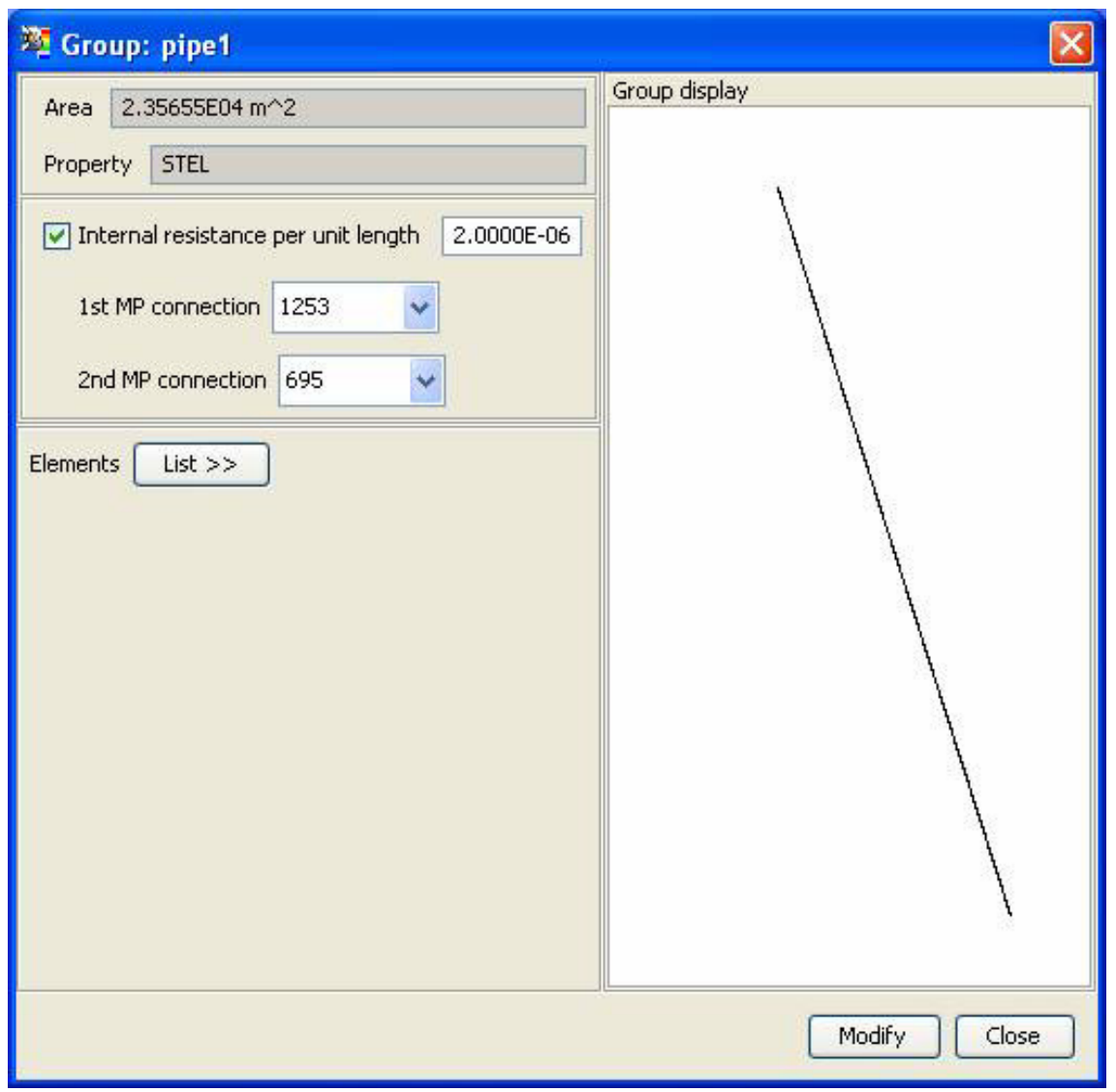

Figure 4: Pipelines properties can be viewed and specified by clicking on the object to display the properties window.

The internal resistance of pipelines and their connections can be specified in a similar way, as shown in Figure 4

\section{Application}

The technology has been used to simulate performance of a number of Oil \& Gas facilities ranging from Jacket type structures with controlled anodes (i.e. diodes) to deepwater applications with subsea facilities and pipelines. In the application presented here the investigation focuses on an offloading buoy and its anchor chains. 
The anchor chains present an interesting CP problem because there is significant attenuation of the current flowing along the chains due the very high internal resistance caused by the chain link to chain link contact. Therefore near the buoy the chain will receive significant current from the anodes but this will drop off significantly within $25-30 \mathrm{~m}$.

The model of the buoy and its anchor chains is shown in Figure 6.

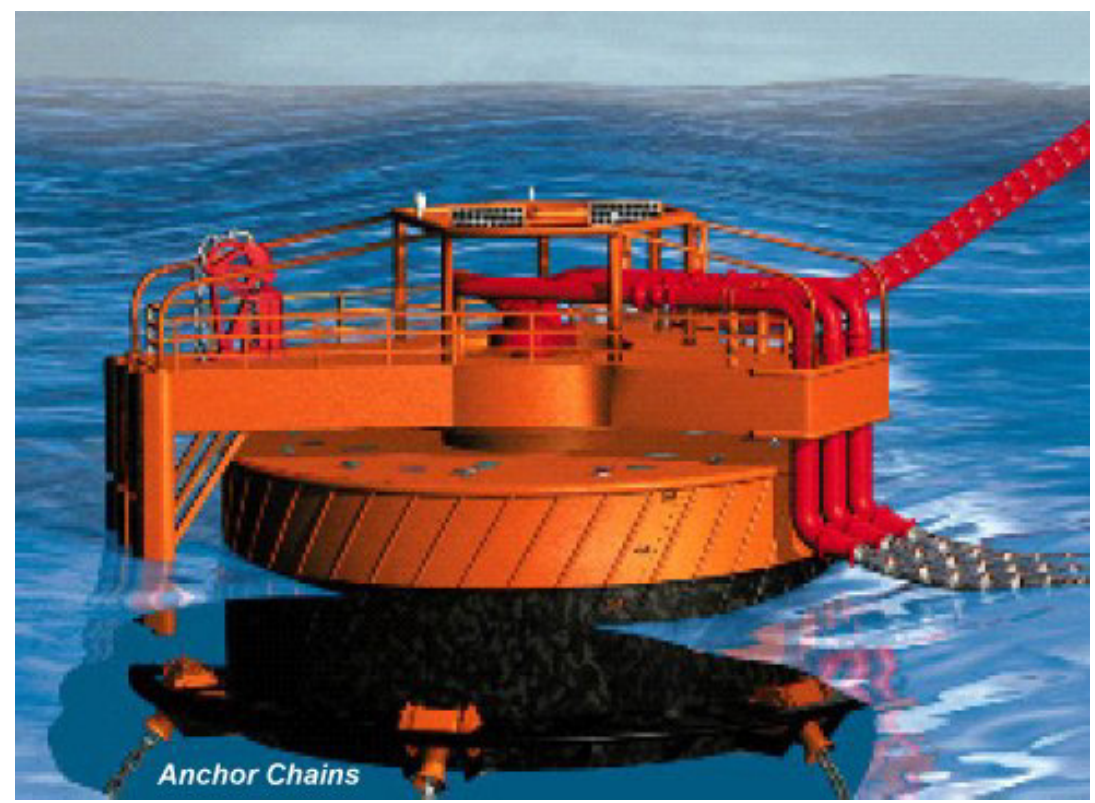

Figure 5: Typical buoy layout with offloading lines and anchors.

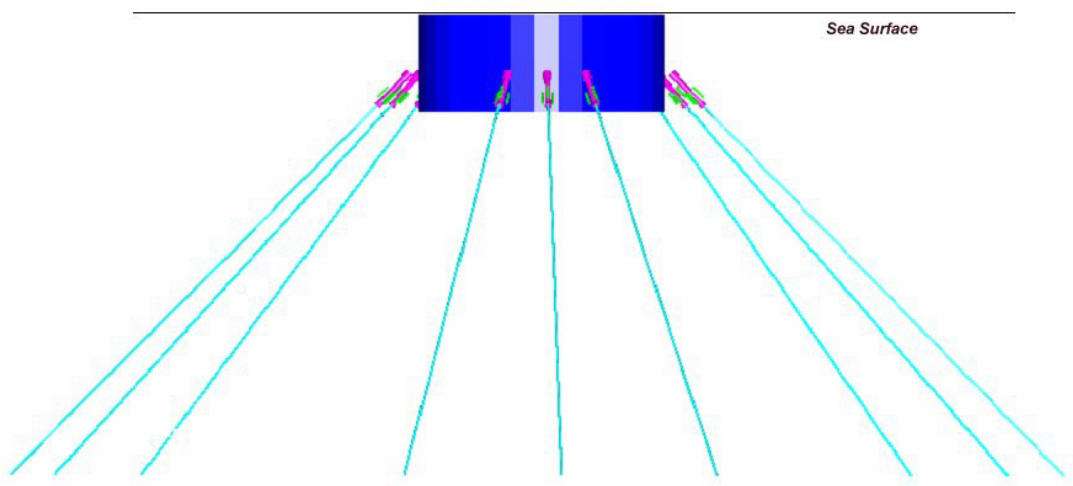

Figure 6: Model of the buoy and the anchor chains. 


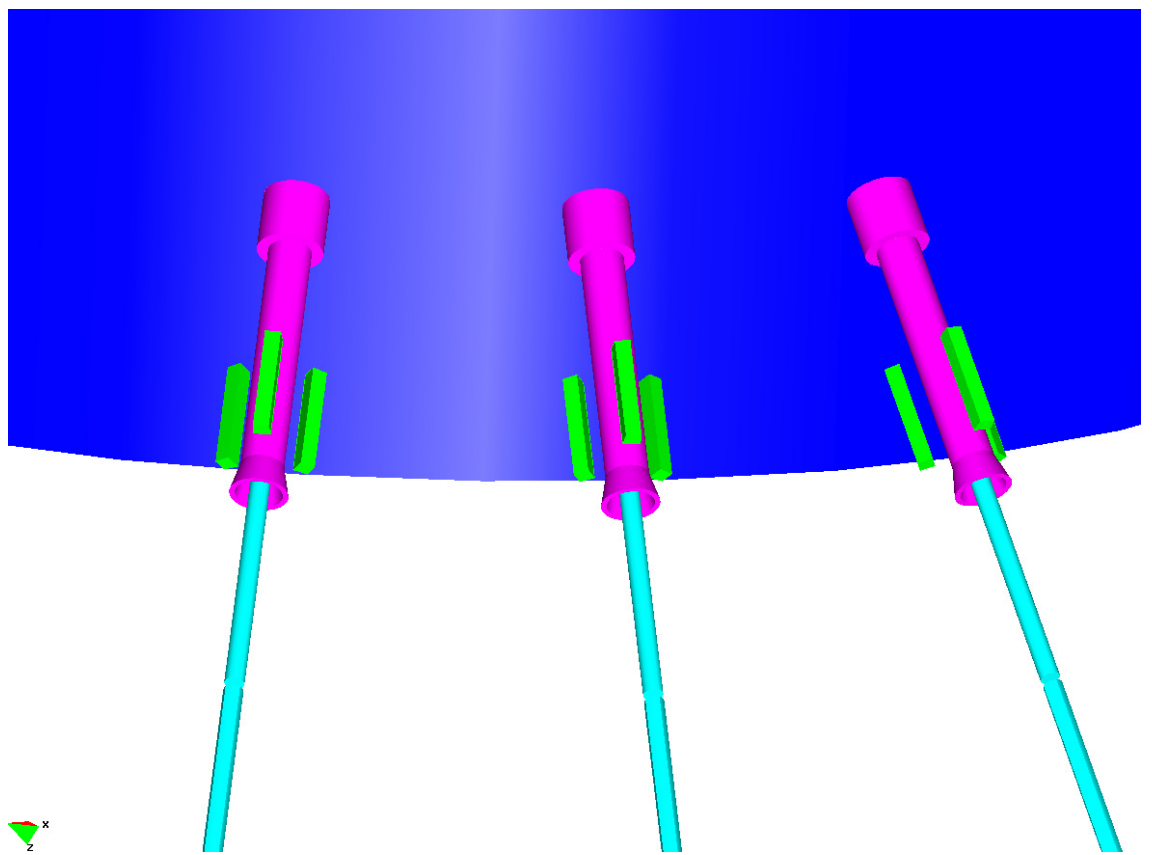

Figure 7: Detailed view of the connections between the buoy and the anchors.

Figure 7 shows the buoy and the anchor connections. The model includes a full 3D model of the connectors (Seen as cylinders in the model) including the internal geometry because the anodes not only need to protect the outer metallic surfaces but also the internal structure. The chains have been electrically connected to the buoy and have a high internal electrical resistance. In the model of the complete system the lines connecting to the FPSO would also be included as well as the FPSO and the subsea systems.

The predicted protection potentials provided by the $\mathrm{CP}$ system is shown in Figure 8 .

The attenuation of the current on the anchors can be clearly seen in figure 9 .

\section{Conclusions}

A method has been demonstrated which allows coupled calculation of the metal voltage drop in pipelines, the potential field in the electrolyte, and the electrode kinetics.

Since the metal voltage drops which occur in practise may be $100 \mathrm{mVolts}$ or more, the determination of such coupled solutions with proper account taken of metal resistance is a very important part of CP design. 


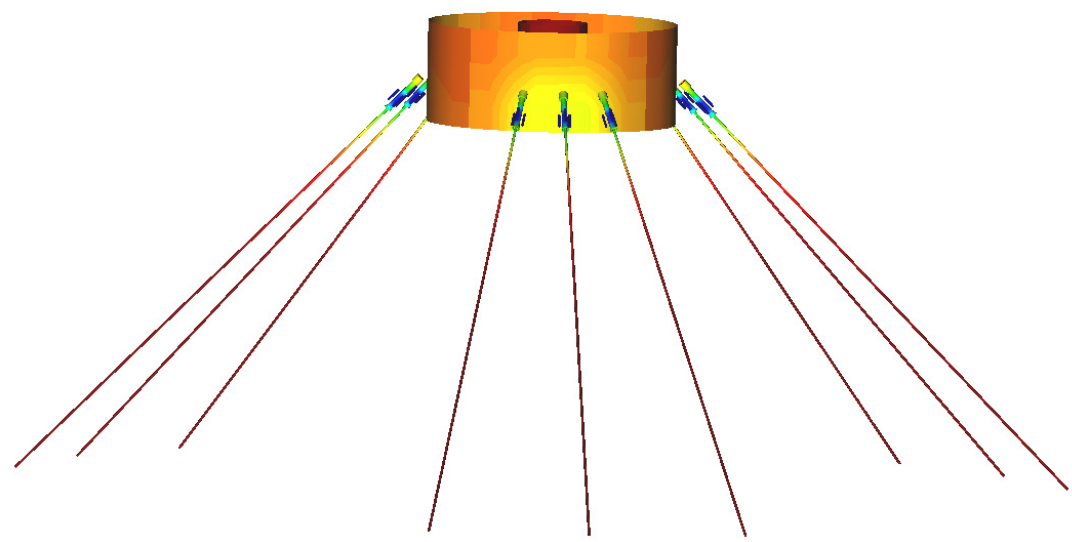

Figure 8: $\quad$ Protection potentials on the buoy and anchors.

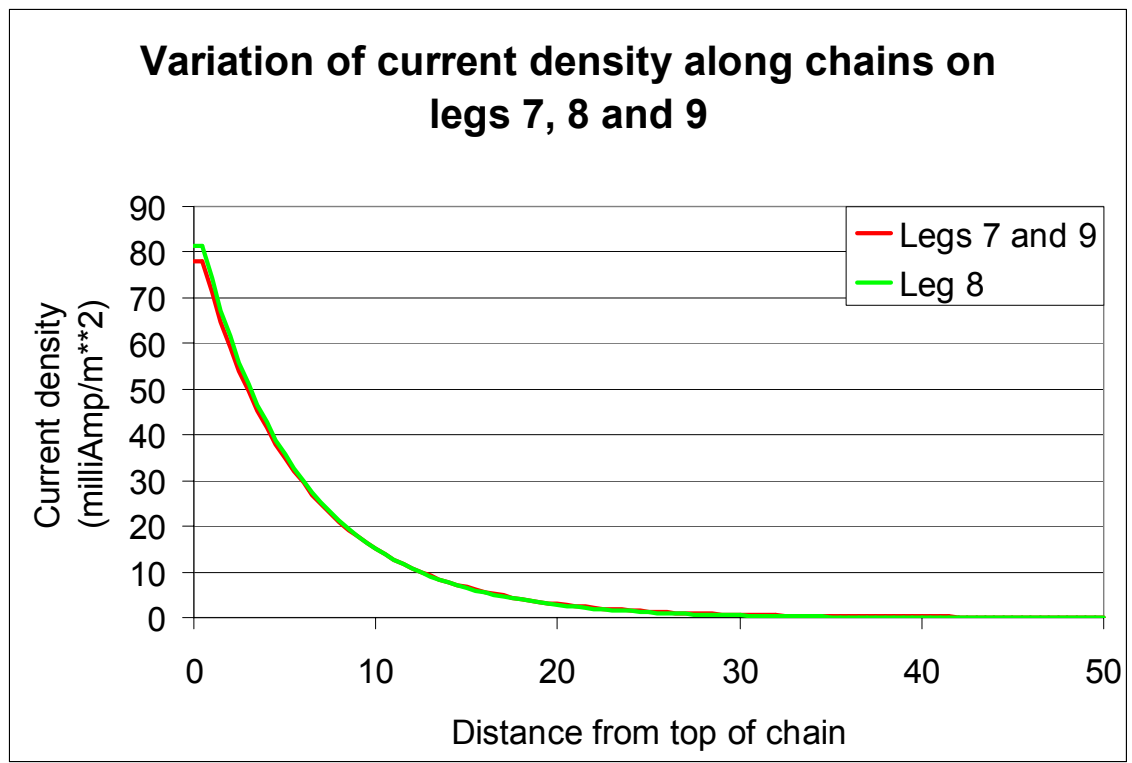

Figure 9: Attenuation of the current density on the anchor with distance from the buoy.

\section{References}

[1] Brebbia C.A, Boundary Element Method for Engineers, Pentech Press. London, 1978. 
[2] Danson, D.J. and Warne, M.A. Current Density/Voltage Calculations Using Boundary Element Techniques, Corrosion/83. 1983.

[3] Thomas, E. D., Lucas, K. E., and Parks, A. R., Verification of Physical Scale Modeling with Shipboard Trails, Corrosion 90, Paper 370, National Association of Corrosion Engineers, Houston, TX, 1990.

[4] V.G. DeGiorgi, A. Kee, E.D. Thomas. Characterization accuracy in modelling of corrosion systems. Boundary Elements XV. Vol. 1: Fluid Flow and Computational Aspect. Editors: C.A. Brebbia, J.J. Rencis. CML Publications, Southampton, 1993.

[5] Adey R A, Santana Diaz E. Computer Simulation of the Interference Between a Ship and Docks Cathodic Protection Systems, Electrocor 2005, Cadiz, Spain. May 2005. 